\title{
Alternativas tecnológicas para el control de Botrytis sp. en mora de castilla (Rubus glaucus)
}

\section{(Technological alternatives for the control of Botrytis sp. In Andean blackberry (Rubus glaucus))}

\author{
Martin Acosta-Ruales, ${ }^{1}$ William Fernando-Viera, ${ }^{2}$ Trevor Jackson, ${ }^{3}$ Wilson Vásquez-Castillo ${ }^{4}$
}

\begin{abstract}
Resumen
El cultivo de mora de castilla (Rubus glaucus) es de mucha importancia para los agricultores ya que deben implementar prácticas culturales que permitan reducir el uso de pesticidas sintéticos. El objetivo de este estudio fue evaluar el efecto de diferentes alternativas tecnológicas para el control de Botrytis sp. en el cultivo de mora de castilla. Los tratamientos fueron 1: Testigo absoluto (silwet + agua), 2: Fungicida tradicional sintético (Carbendazim), 3: Extracto de mirtáceas (Iridium \&), 4: Metabolitos bacterianos (Balus@) y 5: Trichoderma spp. (Tricomix®). Las aplicaciones se realizaron cada 15 días para el producto sintético y cada 8 días los biológicos, orgánicos y el testigo. Se utilizó un diseño de bloques completamente al azar (DBCA) con 3 repeticiones y un total de 10 unidades experimentales. Las variables estudiadas del fruto fueron: incidencia de Botrytis sp. (\%), rendimiento (g/parcela), peso (g), diámetro polar y ecuatorial (mm), firmeza (N), sólidos solubles totales (Brix), acidez (\% ácido cítrico) y pH (log H+). Se realizó un análisis de varianza y prueba de Tukey al 5 \%. A partir de un análisis de incidencia semanal, se identificó que Carbendazim, Trichoderma spp. y extracto mirtáceas tuvieron un control en la incidencia de Botrytis sp. en el fruto de mora de castilla.
\end{abstract}

\section{Palabras clave}

Biocontroladores; Botrytis sp.; Trichoderma spp.; características fisicoquímicas; incidencia.

\begin{abstract}
The Andean blackberry crop (Rubus glaucus) is very important for the farmers, since they must implement cultural practices that allow reducing the use of synthetic pesticides. The objective of the study was to evaluate the effect of different technological alternatives for the control of Botrytis sp. in the blackberry crop. The treatments were: 1: Absolute control (silwet+water), 2: Traditional chemical treatment (Carbendazim), 3: Mirtaceae extract (Iridium $®), 4$ : Bacterial metabolite (Balus $\otimes$ ) and 5: Trichoderma spp. (Tricomix $®)$. The applications were carried out every 15 days for the synthetic product and every 8 days for the biologicals, organic and the control. A randomized complete block design (RCBD) was used with 3 repetitions and Tukey test at $5 \%$ were made. The studied variables were botrytis incidence (\%), yield (g/plot), weight (g), polar and equatorial diameter (mm), firmness ( $N)$, total soluble solids (Brix), titratable acidity (\% citric acid) and $\mathrm{pH}\left(\log \mathrm{H}^{+}\right)$. Throughout a weekly incidence analysis, it was possibly to identify that the Carbendazim, Trichoderma spp. and mirtaceae extract had a better control in the incidence of Botrytis sp. in the blackberry fruit.
\end{abstract}

\section{Keywords}

Biocontrollers; Botrytis sp.; Trichoderma spp.; physicochemical characteristics; incidence.

\section{Introducción}

La mora de castilla (Rubus glaucus) es uno de los frutales andinos de importancia en el Ecuador. Dentro del país, se han reportado un total de 21 especies de Rubus, entre las más reconocidas están la mora de Castilla, debido a su importancia comercial (Delgado, 2012). Esta fruta se cultiva en todas las provincias de los Andes ecuatorianos, alcanza una superficie cultivada de 5247 ha (SI- 
NAGAP, 2016). La extensión cultivada está manejada por alrededor de 15000 pequeños y medianos productores de la Sierra, los que poseen un nivel tecnológico bajo que requiere mejoramientos a través de programas integrales, manejo fitosanitario y mejoramiento genético (Vásquez, Pupiales, Viteri, Sotomayor, Feican, Campaña y Viera, 2019). La provincia de Tungurahua es la principal productora con un $42 \%$ de superficie plantada, le sigue Chimborazo con el $25 \%$ (MAGAP, 2012).

Una de las enfermedades que ocasiona grandes pérdidas económicas y es considerada como uno de los principales problemas fitosanitarios es la pudrición del fruto, moho gris o pudrición blanda, ocasionado por el hongo Botrytis cinerea (Quinatoa, 2015). Entre las afectaciones más importantes se encuentran la reducción del rendimiento, necrosis del fruto, momificación, quemaduras de las inflorescencias y pudrición del fruto (DANE, 2013).

La afectación por plagas y enfermedades en el cultivo de mora de castilla es la principal limitante. Por esta razón, la implementación del Manejo Integrado de Plagas (MIP) es una prioridad, cuyos componentes son el material de siembra, las labores culturales, plan de nutrición de la planta, uso adecuado de plaguicidas, que permitan el control preventivo de plagas y enfermedades (Jiménez, 2009).

El hongo Botrytis cinerea infecta al cultivo en cualquier estado del desarrollo del fruto y a cualquier parte de la planta, como a los tallos, flores, hojas y frutos (Viteri, et al., 2016). De acuerdo a los estudios que se realizaron en las provincias de Tungurahua, Cotopaxi y Bolívar el porcentaje de daño de esta enfermedad al cultivo puede alcanzar hasta el 91.8 \% (Villares, Martínez, Viteri, Viera, Jácome, Ayala y Noboa, 2016).

Durante los últimos años se han desarrollado métodos de control de plagas en los cultivos. Entre los cuales está, el método cultural que consiste en la aplicación de buenas prácticas agrícolas, una adecuada densidad de siembra, podas y fertilización, considerando las necesidades del cultivo (Restrepo, Peñaranda, Jiménez, Galindo, Rivero, Jiménez y Guerrero, 2012). Otro método es el orgánico y consiste en el uso de productos a base de extractos vegetales, que estimulan las neuronas de insectos afectando adversamente al desarrollo de los mismos (Ramón y Rodas, 2007). El biológico que utiliza biocontroladores de plagas y enfermedades. Uno de ellos Eutiliza hongos como Penicillium spp., Trichoderma spp., Gliocadium spp. (Capelo y Roche, 2008). El control que utiliza moléculas de productos sintéticos, es la medida aplicada por la mayoría de los productores, entre los que encontramos el carbendazim (Cardona, García, Barrera, Quejada, Rodríguez y Arroyave, 2014). El genético, a través del uso de variedades resistentes a ciertas plagas y enfermedades y de alta productividad (Jiménez, 2009)

La producción convencional de los cultivos puede ocasionar diferentes efectos negativos como es el aumento de acidez de los suelos, disminución de la fertilidad y reducción de fauna nativa. A nivel del fruto se ha identificado alteraciones en las características fisicoquímicas, olor y sabor (Guevara, 2016). Debido a esto se diseñaron nuevas tecnologías para la implementación de la agricultura limpia y orgánica, basada en el uso de variedades mejoradas, controladores biológicos y fertilizantes de origen natural, esto, con la finalidad de reducir el uso de insumos de origen sintético, proteger la salud de las personas y del ambiente (Martínez, Bello y Castellanos, 2012; Marco Brown y Reyes Gil, 2003).

En los últimos años, la utilización de controladores biológicos ha ido creciendo debido a la efectividad para controlar a fitopatógenos y proteger el ambiente (Van Driesche, Hoddle, y Center, 2007). El uso de Bacilus thuringiensis y Trichoderma harzanium son varios ejemplos en el control de enfermedades (Naher, Yusuf, Ismail y Hossain, 2014). Trichoderma spp. es un hongo saprófito y anaerobio facultativo (realiza la respiración con o sin presencia de oxígeno), pertene- 
ciente a la familia Monilia y género Trichoderma. Generalmente se lo encuentra en suelos con gran cantidad de materia orgánica (Martínez, Infante y Reyes, 2013). La eficacia de este hongo en el control de enfermedades se debe a que inhibe el crecimiento de ciertos fitopatógenos a través de la producción de metabolitos (Martínez, Infante y Reyes, 2013).

Con este antecedente, el estudio consitió en determinar el efecto de los productos de síntesis, biológicos y orgánicos en la incidencia de Botrytis sp., calidad físicoquímica y rendimiento del fruto de mora de castilla.

\section{Metodología}

El estudio se realizó desde el mes de junio hasta noviembre de 2018 en un total de tiempo de 24 semanas, en la granja experimental de la Universidad de las Américas-UDLA, ubicada a $78^{\circ} 34^{\prime}$ $22 " \mathrm{O}$ de longitud, $0^{\circ} 4^{\prime} 24^{\prime \prime} \mathrm{S}$ de latitud y a una altura de $2720 \mathrm{msnm}$. La temperatura y humedad relativa promedio fue de $14{ }^{\circ} \mathrm{C}$ y $70 \%$, respectivamente, la precipitación anual de $1161.7 \mathrm{~mm}$, el suelo es franco-arenoso y con un pH de 6.84. La evaluación físicoquímica del fruto se realizó en los laboratorios de la UDLA sede Queri, ubicados a una altura de $2831 \mathrm{msnm}, 78^{\circ} 28^{\prime} 14^{\prime \prime} 0$ de longitud y $0^{\circ} 10^{\prime} 10^{\prime \prime} S$ de latitud.

Se utilizó un diseño de bloques completamente al azar (DBCA) con cinco tratamientos y tres repeticiones. Se realizó un análisis de varianza aplicando la prueba de Tukey al 5 \%. Los tratamientos fueron T1: testigo (silwet+agua) con una dosis de $0.15 \mathrm{cc} \mathrm{l}^{-1}, \mathrm{~T} 2$ : producto sintético (Carbendazim) en dosis de $1 \mathrm{cc} \mathrm{l}^{-1}$, T3: producto orgánico (extracto de mirtáceas) con dosis de 3 $\mathrm{cc} \mathrm{l}^{-1}$, T4: microorganismo benéfico (metabolitos bacterianos) con dosis de $8 \mathrm{cc}^{-1}$ y T5: microorganismo benéfico (Trichoderma spp.) con dosis de $0.37 \mathrm{~g} 20 \mathrm{l}^{-1}$. La aplicación del testigo, producto orgánico y microorganismos benéficos fue cada 8 días, mientras que el producto comercial se aplicó cada 15 días.

Los datos meteorológicos desde el mes de junio hasta noviembre se obtuvieron de la estación meteorológica de Nono ubicada dentro de la granja experimental de la UDLA. Se recopilaron los datos diarios de precipitación a las 07, 13 y 19 horas. Se utilizó un data logger (Hobo Pro v2), colocado en las parcelas de mora, para recolectar los valores de temperatura máxima, temperatura mínima y humedad relativa diaria por hora.

Las variables que se estudiaron fueron: incidencia de la enfermedad en el fruto (\%), semanalmente se registró la presencia de la enfermedad en los frutos cosechados el porcentaje estuvo en relación con los frutos sanos. El rendimiento (g/parcela) se evaluó por 6 meses, para esto se consideró el rendimiento de las cosechas semanales de cada tratamiento (parcela neta). La cosecha se hizo en el grado de madurez 3, utilizando un colorímetro Lovibond LC100 (figura 1).

Las variables de calidad física y química del fruto se registraron cada mes y fueron peso del fruto $(\mathrm{g})$, quese determinó tomando al azar de cada tratamiento 10 frutos y al final se calculó la media aritmética. El pH $\left(\log \mathrm{H}^{+}\right)$que se obtuvo por la medición de un extracto producto de la maceración de $15 \mathrm{~g}$ de fruta, con el uso de un potenciómetro. Los SST ( ${ }^{\circ}$ Brix) se midieron a partir de $1 \mathrm{~g}$ de fruta colocado en un refractómetro. La acidez titulable (\% ácido cítrico) se realizó con 30 $\mathrm{g}$ de jugo de mora mezclado con $200 \mathrm{ml}$ de agua destilada, que luego fue titulado con $\mathrm{NaOH} 1 \mathrm{~N}$ hasta llegar a un pH de 8-8.2. El diámetro polar y ecuatorial $(\mathrm{mm})$ se midió con un calibrador para luego calcular la media aritmética. Para la firmeza (N) se tomaron 5 frutos representativos y al azar de cada tratamiento, se midió con un penetrómetro para obtener luego la media aritmética. 
Figura 1. Color de la fruta a cosechar según especificaciones

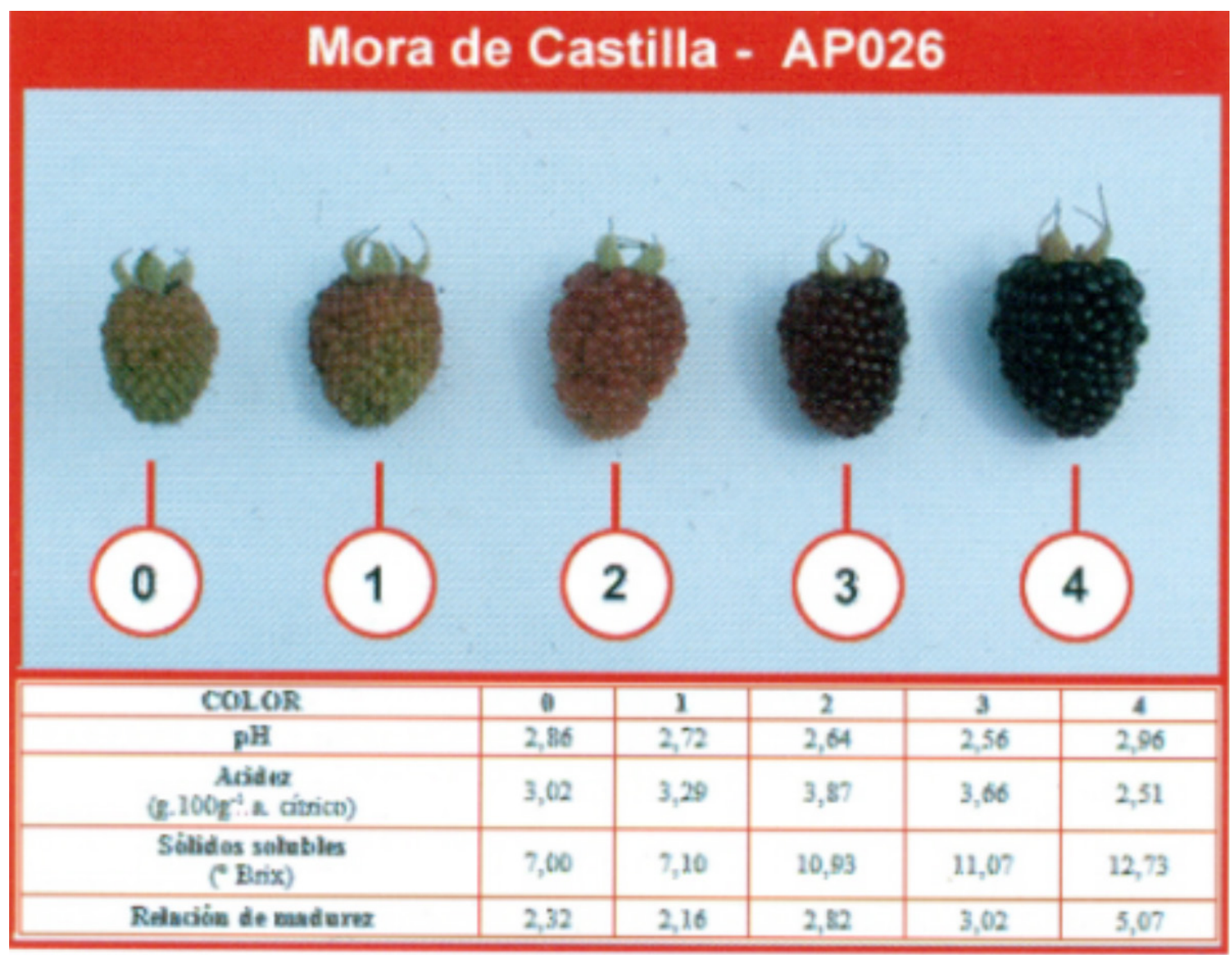

Fuente: (Brito, Montalvo, Freire, Vásquez, Viteri, Martínez y Jácome, 2016)

\section{Resultados y discusión}

Al realizar la evaluación semanal (24) de la incidencia de Botrytis spp. en los frutos de mora, se determinó la existencia de diferencias estadísticas entre los tratamientos en las semanas 28, 41 y 45. En la semana 28 (9-julio-2018) con un valor de p (0.0078), se evidenció que el testigo y las plantas tratadas con metabolitos bacterianos no presentaron la enfermedad, mientras que las plantas tratadas con Trichoderma spp. tienen la mayor incidencia con $10.53 \%$. Esto pudo deberse a que no sucedió la esporulación de Trichoderma spp. por las condiciones ambientales secas, ya que el hongo necesita humedades entre 70 \% y80 \% para su crecimiento (Vásquez J., 2010).

Por otro lado, se evidencia que en las semanas 41 (08-octubre-18) con un valor de $p$ (0.0349) y 45 (05-noviembre-18) con valor de $\mathrm{p}$ (0.0389), los productos metabolitos bacterianos, mirtáceas, carbendazim y Trichoderma spp. tuvieron un control similar sobre la enfermedad; siendo iguales estadísticamente entre ellos, pero diferentes al testigo. El comienzo de las precipitaciones en el mes de octubre (total $4.60 \mathrm{~mm}$ ) y las temperaturas (promedio de $12.81^{\circ} \mathrm{C}$ ) pudieron haber favorecido en la incidencia de Botrytis $\mathrm{sp}$. 
Tabla 1. Incidencia de Botrytis sp. en los frutos de mora de castilla después de la aplicación de productos de diferentes orígenes en los Andes del Ecuador

\begin{tabular}{|l|l|l|l|l|}
\hline \multirow{2}{*}{\multicolumn{1}{|c|}{ Tratamientos }} & \multicolumn{4}{c|}{ Incidencia (\%) semanas } \\
\cline { 2 - 5 } & \multicolumn{1}{|c|}{$\mathbf{2 8}(\mathbf{0 9 - 0 7 - 1 8 )}$} & \multicolumn{1}{|c|}{$\mathbf{3 3}(\mathbf{1 3 - 0 8 - 1 8 )}$} & \multicolumn{1}{|c|}{$\mathbf{( 0 8 - 1 0 - 1 8 )}$} & \multicolumn{1}{|c|}{$\mathbf{4 5}$ (05-11-18) } \\
\hline 5. Trichoderma spp. & $10.53 \pm 6 \mathrm{~b}$ & $12.70 \pm 13.2$ & $0.93 \pm 0.8 \mathrm{ab}$ & $2.73 \pm 3.2 \mathrm{ab}$ \\
\hline 3. Mirtáceas & $3.93 \pm 3.3 \mathrm{ab}$ & $6.03 \pm 5.2$ & $1.43 \pm 1.2 \mathrm{ab}$ & $0 \pm 0 \mathrm{a}$ \\
\hline 2. Carbendazim & $4.57 \pm 3.1 \mathrm{ab}$ & $8.90 \pm 4.4$ & $2.67 \pm 2.5 \mathrm{ab}$ & $5.63 \pm 1.8 \mathrm{ab}$ \\
\hline 1. Testigo & $0 \pm 0 \mathrm{a}$ & $2.17 \pm 3.7$ & $5.13 \pm 1.2 \mathrm{~b}$ & $8.80 \pm 5.4 \mathrm{~b}$ \\
\hline 4. Metabolitos bacterianos & $0 \pm 0 \mathrm{a}$ & $17.50 \pm 13.7$ & $0 \pm 0 \mathrm{a}$ & $3.27 \pm 2.9 \mathrm{ab}$ \\
\hline
\end{tabular}

Promedios seguidos de la misma letra, son estadísticamente iguales (Tukey $5 \%$ )

En general, el uso del producto de origen orgánico prácticamente fue muy similares al de origen sintético en el control de la enfermedad, sin embargo, fueron mejores en comparación con el controlador biológico como Trichoderma spp. Este resultado concuerda con lo reportado por Alencastro (2011), quien indica que los productos con mayor efectividad en el control del hongo Botrytis cinerea o moho gris son los benzimidazoles (Carbendazim) y dicarboximidas, esto se debe que tienen un mayor espectro de acción. De igual manera Tello, Oña, Jarrín y Viera (2018) afirman que existe una inhibición del 100 \% en el crecimiento micelar con la aplicación de carbendazim.

Hincapié, Saldarríaga y Díaz (2016) indican que un monitoreo de las condiciones climáticas, severedidad de las enfermedades y la aplicación de productos sintéticos en rotación con productos biólogicos es uno de los mejores tratamientos en el control de Botrytis cinerea.

Además (Martínez, Villacís, Viera, Jacome, Espín, León y Rita, 2019) pascual determinan la necesidad de incluir Trichoderma spp. en el manejo limpio ya que las cepas colonizan las raíces protegiéndolas de cualquier patógeno. De esta forma las ramas en producción se verán sanas al igual que los frutos.

Para la variable peso del fruto, el efecto de los tratamientos no influyó por lo que no hubo diferencias estadísticas al $5 \%$, sin embargo, existieron fluctuaciones del peso ya que hubo variaciones entre 2.73 y $6.72 \mathrm{~g}$ (tabla 2 ).

Tabla 2. Peso del fruto de mora de castilla después de la aplicación de productos de diferentes orígenes en los Andes del Ecuador

\begin{tabular}{|l|l|l|l|l|l|l|}
\hline \multirow{2}{*}{\multicolumn{1}{|c|}{ Tratamientos }} & \multicolumn{7}{c|}{ Peso fruto $(\mathrm{g})$} \\
\cline { 2 - 7 } & Junio & \multicolumn{1}{c|}{ Julio } & \multicolumn{1}{c|}{ Agosto } & \multicolumn{1}{c|}{ Septiembre } & \multicolumn{1}{c|}{ Octubre } & \multicolumn{1}{c|}{ Noviembre } \\
\hline Metabolitos bacterianos & $3.97 \pm 2.5$ & $3.88 \pm 0.8$ & $5.05 \pm 1.6$ & $3.25 \pm 3.3$ & $4.95 \pm 0.8$ & $4.01 \pm 0.6$ \\
\hline Carbendazim & $4.75 \pm 2.4$ & $4.38 \pm 0.5$ & $5.73 \pm 1$ & $5.58 \pm 1$ & $3.60 \pm 0.9$ & $4.43 \pm 0.9$ \\
\hline Mirtáceas & $4.80 \pm 0.7$ & $4.56 \pm 0.8$ & $5.63 \pm 0.6$ & $5.55 \pm 0.5$ & $4.44 \pm 1.4$ & $4.57 \pm 1$ \\
\hline Testigo & $5.69 \pm 2.9$ & $4.50 \pm 0.9$ & $4.57 \pm 2.4$ & $5.57 \pm 1.1$ & $5.02 \pm 1.6$ & $4.53 \pm 0.4$ \\
\hline Trichoderma spp & $6.72 \pm 0.4$ & $3.66 \pm 1.8$ & $5.05 \pm 3.1$ & $4.51 \pm 0.6$ & $4.72 \pm 1.1$ & $2.73 \pm 2.5$ \\
\hline
\end{tabular}

Promedios seguidos de la misma letra, son estadísticamente iguales (Tukey 5 \%)

De acuerdo con (Merchán, 2014) la fluctuación de peso se debió a la eficiencia de la cepa de Trichoderma, ya que este hongo influye directamente en la asimilación de nutrientes en la planta que a su vez pudo generar frutos de mayor o menor tamaño. Por otro lado, Sánchez, Vi- 
llares, Niño y Ruilova (2018) indican que estas diferencias o fluctuaciones en el tamaño de los frutos pueden estar influenciadas por las temperaturas bajas. Otra de las posibilidades para las variaciones en peso del fruto puede ser el manejo agronómico del cultivo, especialmente el riego, ya que aporta con un mayor número de frutos y de mayor calibre (Rodríguez, 2018).

En las variables firmeza del fruto y solidos solubles totales (tabla 3) existieron diferencias estadísticas al $5 \%$ en el mes de septiembre (semana 39), siendo el testigo el que presentó frutos con mayor firmeza (1.59 N). Mientras que la mayor cantidad de SST alcanzó los tratamientos en los que se aplicó Trichoderma, el testigo y metabolitos bacterianos. Por otro lado, en las evaluaciones realizadas entre los meses de junio a noviembre, no existió efecto de los tratamientos en las variables antes indicadas. Las condiciones ambientales predominantes durante el período de evaluación fueron muy cambiantes en la zona de estudio, favoreciendo en unos casos el crecimiento del patógeno y/o inhibiendo el desarrollo del microorganismo benéfico para controlar la enfermedad. Esto se evidenció, puesto que las lluvias se presentaron en septiembre (1.21 $\mathrm{mm}$ ) y temperaturas de $24.68{ }^{\circ} \mathrm{C}$ en promedio, condiciones ambientales diferentes a las de los meses secos (junio a agosto) que tuvieron una precipitación promedio de $0.15 \mathrm{~mm}$.

Tabla 3. Firmeza del fruto (N) y sólidos solubles totales ( $\left.{ }^{\circ} \mathrm{Brix}\right)$ de mora de castilla después de la aplicación de productos de diferentes orígenes en los Andes del Ecuador

\begin{tabular}{|l|l|l|}
\hline \multicolumn{1}{|c|}{ Tratamientos } & \multicolumn{1}{|c|}{ Firmeza (N) } & \multicolumn{1}{c|}{ SST ( ${ }^{\circ}$ Brix) } \\
\cline { 2 - 3 } & \multicolumn{1}{|c|}{ Septiembre } & \multicolumn{1}{c|}{ Septiembre } \\
\hline Metabolitos bacterianos & $0.81 \pm 0.4 \mathrm{a}$ & $8.23 \pm 0.5 \mathrm{~b}$ \\
\hline Trichoderma spp. & $0.93 \pm 0.3 \mathrm{ab}$ & $9.17 \pm 0.7 \mathrm{~b}$ \\
\hline Mirtáceas & $1.30 \pm 0.4 \mathrm{ab}$ & $8.00 \pm 0.9 \mathrm{a}$ \\
\hline Carbendazim & $1.39 \pm 0.5 \mathrm{ab}$ & $9.03 \pm 0.5 \mathrm{ab}$ \\
\hline Testigo & $1.59 \pm 0.6 \mathrm{~b}$ & $9.20 \pm 0.8 \mathrm{~b}$ \\
\hline
\end{tabular}

Promedios seguidos de la misma letra, son estadísticamente iguales (Tukey $5 \%$ )

La disminución de la firmeza en los frutos con la aplicación de metabolitos bacterianos pudo implicar que, debido a la precipitación anteriormente mencionada en el mes de septiembre, generó una susceptibilidad en el ablandamiento del fruto causado por Botrytis. Esto pudo activar el proceso de degradación de la pared celular, dando como resultado esta disminución en la firmeza (Merchán, 2014).

Otra de las razones de este hecho es que a medida que aumenta el estado de madurez de los frutos, las paredes celulares se vuelven más débiles y empiezan a perder agua debido al comportamiento de la enzima hidrolasa. Es por esto que se debe realizar la cosecha con un grado de madurez óptimo del fruto, ya que la pared celular de un fruto con madurez alta tiende a degradarse más rápido que un fruto con madurez temprana (Deaquiz y Brigitte, 2015).

En cuanto a la variable de SST, existieron diferencias significativas al 5 \% únicamente en el mes de septiembre (tabla 3), durante este mes alcanzaron un promedio de $8.72^{\circ} \mathrm{Brix}$. Durante la semana 39 y la semana 37, correspondiente al mes de septiembre, se registró una de las temperaturas más altas $\left(26.77^{\circ} \mathrm{C}\right.$ ) y una nubosidad de cinco octavos (fracción del cielo cubierta de nubes) correspondientemente. Las altas temperatura y la nubosidad que se presentaron posiblemente afectó a la formación de azúcares solubles en los frutos. De acuerdo a Deaquiz y Brigitte (2015), se mencionó que la variedad Rubus glaucus tiene una gran cantidad de SST en compa- 
ración con el resto de las variedades existentes. Esta variedad puede registrar hasta $11.3^{\circ} \mathrm{Brix}$ de acuerdo al piso altitudinal que se encuentre y a las diferentes características climáticas. En otro estudio, se obtuvieron valores promedios de ${ }^{\circ} B r i x$ menores a los reportados anteriormente, lo que confirma que la alta luminosidad o insolaciones están directamente relacionadas con la formación de SST en las primeras etapas de fructificación (Grijalba, Calderón y Pérez, 2010).

En cuanto a la variable de acidez (ácido cítrico), los productos que se aplicaron no influenciaron en la acidez del fruto de mora de castilla, puesto que no existieron diferencias estadísticas entre los tratamientos. Como se observa en la tabla 4. En general, la mayor acidez fue de 2.16 y la menor fue de 1.28 .

Los valores de acidez obtenidos en este estudio están acordes con lo reportado por Iza, Rojas y Arguello (2016), quienes indican que la acidez titulable de la mora esta entre 2.20 a 2.47 $\%$. De igual manera, identificaron que la disminución de la acidez se debe al grado de madurez de los frutos, ya que, a mayor grado de madurez, la tasa de respiración y el contenido de azúcares solubles aumentan.

Tabla 4. Acidez y pH del fruto de mora de castilla después de la aplicación de productos de diferentes orígenes en los Andes del Ecuador

\begin{tabular}{|l|l|l|}
\hline \multicolumn{1}{|c|}{ Tratamientos } & \multicolumn{1}{c|}{ Acidez (\% ácido cítrico) } & \multicolumn{1}{c|}{ pH } \\
\hline Metabolitos bacterianos & $1.28 \pm 0.8$ & $1.31 \pm 0.2$ \\
\hline Trichoderma spp. & $2.16 \pm 0.4$ & $2.23 \pm 0.1$ \\
\hline Mirtáceas & $1.50 \pm 0.5$ & $1.46 \pm 0.1$ \\
\hline Carbendazim & $1.79 \pm 0.4$ & $1.66 \pm 0.2$ \\
\hline Testigo & $1.96 \pm 0.4$ & $1.82 \pm 0.1$ \\
\hline Promedio & 1.73 & 1.70 \\
\hline
\end{tabular}

Para la variable del pH del fruto no se presentaron diferencias significativas al $5 \%$, sin embargo, el mayor valor (2.23) se obtuvo al realizar el control de Botrytis sp. con el uso de Trichoderma spp. y el valor mínimo con los metabolitos Bacterianos (1.31). Ayala, Valenzuela y Bohórquez (2013) registraron ligeros cambios en el pH pero con un rango promedio de valores entre 2.64 y 2.88; estas variaciones en el pH están relacionadas con el grado de madurez del fruto. El incremento o disminución del pH en los frutos está directamente relacionado con las condiciones climáticas, ya que a mayor temperatura en los primeros días del manejo poscosecha puede existir variación en la composición química del fruto (Iza, Rojas y Arguello, 2016).

\section{Conclusiones y recomendaciones}

Al inicio del experimento los efectos para controlar Botrytis sp. no fueron tan significativos como al finalizar el estudio ya que durante los meses del período seco (junio-agosto) no existieron las condiciones ambientales óptimas para el desarrollo del hongo. Sin embargo, en los meses en que inicio las lluvias (septiembre y noviembre), los productos con mejores resultados para controlar la enfermedad fueron Trichoderma spp., extracto de mirtáceas y Carbendazim.

Los diferentes tratamientos aplicados en el estudio no afectaron a la calidad física y química de los frutos de mora de castilla.

Es recomendable, repetir el estudio en el período lluvioso (óptimo para el desarrollo del patógeno) para confirmar los resultados de esta investigación. 


\section{Agradecimientos}

Los autores agradecen a AgReserach por la asistencia técnica y al Gobierno de Nueva Zelandia (MFAT New Zealand) por el financiamiento brindado. Este artículo forma parte del II Simposio Internacional de Producción Integrada de Frutas 2019.

\section{Bibliografía}

Alencastro, L. (2011). Alternativas ecológicas para el control del moho gris (Botrytis cinerea) en mora de castilla (Rubus glaucus Benth). Salgolqui: Escuela Poltécnica del Ejército. Recuperado el 12 de septiembre de 2018, de http://repositorio.espe.edu.ec/xmlui/bitstream/handle/21000/4593/TESPE-IASA\%20I-004570.pdf?sequence=1\&isAllowed $=y$

Ayala, L., Valenzuela, C., y Bohórquez, Y. (2013). Variables determinantes de la madurez comercial en la mora de castilla (Rubus glaucus Benth). Tolima: Scientia Agrolimentaria. Recuperado el 19 de Ooctubre de 2018, de http://revistas.ut.edu.co/index.php/scientiaagro/article/view/29

Brito, B., Montalvo, D., Freire, V., Vásquez, W., Viteri, P., Martínez, A. y Jácome, R. (2016). Calidad en la cosecha, poscosecha y comercialización. En D. Galarza, S. Garcés, J. Velásquez, V. Sánchez, y J. Zambrano, El cultivo de la mora en el Ecuador, 137-163. Quito, Ecuador: Instituto Nacional de Investigaciones Agropecuarias (INIAP).

Capelo, G. y Roche, J. (2008). Evaluación de 10 fungicidas en el control de Botrytis cinerea Pers.: Fr. en el cultivo de Fresa (Fragaria virginiana Var. Diamante) a nivel de laboratorio. Cuenca, Ecuador: Universidad de Cuenca. Recuperado el 25 de Agosto de 2018, de http://dspace.ucuenca.edu. ec/bitstream/123456789/3035/1/tag281.pdf

Cardona, E., García, E., Barrera, E., Quejada, O., Rodríguez, H., y Arroyave, I. (2014). Manual Técnico del Cultivo de Mora Bajo Buenas Prácticas Agrícolas. Medelín: Gobernación de Antioquia. Recuperado el 18 de julio de 2018, de https://conectarural.org/sitio/material/manual-te\%CC\%81cnico-delcultivo-de-mora-bajo-buenas-pra\%CC\%81cticas-agri\%CC\%81colas

DANE. (2013). El cultivo de la mora de Castilla (Rubus glaucus Benth) frutal de clima frío moderado, con propiedades curativas para la salud humana. Bogotá: SIPSA. Recuperado el 15 de abril de 2018, de https://www.dane.gov.co/files/investigaciones/agropecuario/sipsa/insumos_factores_de_ produccion_nov_2013.pdf

Deaquiz, Y., y Brigitte, M. (2015). Parámetros Poscosecha en dos Materiales de Mora (Rubus Glaucus Benth Y Rubus Alpinus Macfad). Revista Cultura Científica, 16-25. Recuperado el 21 de octubre de 2018, de https://www.researchgate.net/publication/304999743_Parametros_Poscosecha_ en_dos_Materiales_de_Mora_Rubus_Glaucus_Benth_Y_Rubus_Alpinus_Macfad

Delgado, F. (2012). Manejo orgánico del cultivo de mora (Rubus sp.). Cuenca, Ecuador: Universidad de Cuenca.

Grijalba, C., Calderón, L., y Pérez, M. (2010). Rendimiento y calidad de la fruta en mora de castilla (Rubus glaucus Benth), con y sin espinas, cultivada en campo abierto en Cajicá (Cundinamarca, Colombia). Facultad de Ciencias Básicas, 25-41. Recuperado el 17 de junio de 2018, de https:// www.researchgate.net/profile/Maria_Perez-Trujillo/publication/277264501_Rendimiento_y_calidad_de_la_fruta_en_mora_de_castilla_rubus_glaucus_benth_con_y_sin_espinas_cultivada_en_campo_abierto_en_Cajica_Cundinamarca_Colombia/links/55c8926408aeca747d

Guevara, J. (2016). Evaluación del efecto antifúngico de quitosano para el control de podredumbres en mora de castilla (Rubus glaucus) durante el período de poscosecha. Quito, Ecuador: Escuela Politécnica Nacional. Recuperado el 27 de Julio de 2018, de http://bibdigital.epn.edu.ec/handle/15000/16516

Hincapié, O., Saldarríaga, A., y Díaz, C. (2016). Biological, botanical and chemical alternatives for the control of blackberry (Rubus glaucus Benth) diseases. Revista Facultad Nacional de Agrono- 
mía, 70 (2): 8169-8176. Recuperado el 13 de Abril de 2018, de http://www.scielo.org.co/scielo. php?script=sci_arttext\&pid=S0304-28472017000208169

Iza, F., Rojas, X., y Arguello, Y. (2016). Línea base de la calidad de la mora de castilla (Rubus glaucus) en su cadena alimentaria. Enfoque UTE. doi: https://doi.org/10.29019/enfoqueute.v7n3.107

Jiménez, E. (2009). Métodos de control de plagas. Managua: Universidad Nacional Agraria.

MAGAP. (2012). Reporte de Resultados del Censo Provincial Completo en excel (Todas las provincias). Recuperado el 03 de octubre de 2017, de http://sinagap.agricultura.gob.ec/resultados-provincialeshttp://sinagap.agricultura.gob.ec/resultados-provinciales

Marco Brown, L., y Reyes Gil, E. (2003). Tecnologías limpias aplicadas a la agricultura. Interciencia, 28 (5): 252-258. Recuperado el 01 de abril de 2018, de http://www.scielo.org.ve/scielo.php?pid=S0378$18442003000500002 \&$ script $=$ sci_abstract

Martinez, A., Villacís, L., Viera, W., Jacome, R., Espín, M., León, O. y Santana, R. (2019). Evaluación de nuevas tecnologías de producción limpia de la mora de castilla (Rubus glaucus Benth), en la zona Andina de Ecuador, para un buen vivir de los fruticultores. Journal of the Selva Andina Biosphere (7): 63-70. Recuperado el 30 de septiembre de 2019, de https://www.researchgate.net/publication/334083974_Evaluacion_de_nuevas_tecnologias_de_produccion_limpia_de_la_mora_de_castilla_Rubus_glaucus_Benth_en_la_zona_Andina_de_Ecuador_para_un_buen_vivir_de_los_fruticultores

Martinez, B., Infante, D. y Reyes, Y. (2013). Trichoderma spp. y su función en el control de plagas en los cultivos. Revista de Protección Vegetal, 28 (1), 1-11. Recuperado el 25 de septiembre de 2019, de http://scielo.sld.cu/scielo.php?script=sci_arttext\&pid=\$1010-27522013000100001\&lng=es\&tlng=es

Martínez, L., Bello, P. y Castellanos, O. (2012). Sostenibilidad y desarrollo: el valor agregado de la agricultura orgánica. Bogotá: Universidad Nacional de Colombia. Recuperado el 10 de Junio de 2018, de https://www.researchgate.net/publication/306091337_Sostenibilidad_y_Desarrollo_el_valor_agregado_de_la_agricultura_organica

Merchán, J., Ferrucho, R., y Álvarez, J. (2014). Efecto de dos cepas de Trichoderma en el control de Botrytis cinerea y la calidad del fruto en fresa (Fragaria sp.). Revista Colombiana de Ciencias Hortícolas, 8 (1): 44-56.

Naher, L., Yusuf, U., Ismail, A. y Hossain, K. (2014). TRICHODERMA SPP.: A BIOCONTROL AGENT FOR SUSTAINABLE MANAGEMENT OF PLANT DISEASES. Pakistani Journal of Botany, 46 (4): 1489-1493. Recuperado el 16 de marzo de 2018, de https://www.researchgate.net/publication/281736621 Trichoderma_spp_A_biocontrol_agent_for_sustainable_management_of_plant_diseases

Quinatoa, N. (2015). Evaluación del control de botrytis (Botrytis cinerea) en el cultivo de mora (Rubus glaucus Benth) mediante el uso de Trichoderma y EMAS en la comunidad de Misquillí de la parroquía Santa Rosa, provincia de Tungurahua. Ambato, Ecuador: Universidad Técnica de Ambato.

Rabón, W. (2001). Desarrollo de un manejo integrado de Botrytis cinerea en lechuga lisa (Lactuca sativa) bajo condiciones de invernadero en la Sabana de Bogotá. Bogotá, Colombia: Universidad Nacional.

Ramón, V., y Rodas, F. (2007). El control orgánico de plagas y enfermedades de los cultivos y la fertilización natural del suelo. Lima: GROEM HART. Recuperado el 23 de junio de 2018, de http://caminosostenible.org/wp-content/uploads/BIBLIOTECA/guia_contol_organico_plagas.pdf

Restrepo, J., Peñaranda, E., Jimenez, A., Galindo, J., Rivero, M., Jimenez, Y., y Guerrero, M. (2012). Manejo fitosanitario del cultivo de hortalizas. Bogotá: Instituto Colombiano Agropecuario. Recuperado el 10 de abril de 2018, de https://www.ica.gov.co/getattachment/bb883b42-80da-4ae5-851f4db05edf581b/Manejo-fitosanitario-del-cultivo-de-hortalizas.aspx

Rodríguez, I. (2018). Aplicación de nutrientes foliares en los estados fenológicos del cultivo de mora (Rubus glaucus Benth) en la granja experimental Píllaro. Ambato: Universidad Técnica de Ambato. Recuperado el 19 de Mayo de 2018, de http://repositorio.uta.edu.ec/jspui/handle/123456789/27128

Sánchez, J., Villares, M., Niño, Z. y Ruilova, M. (2018). Effect of the altitudinal floor on the quality of mora (Rubus glaucus benth) in the interand region of Ecuador. Idesia (Arica), 209-215. Recuperado el 
17 de Octubre de 2018, de https://scielo.conicyt.cl/scielo.php?script=sci_abstract\&pid=S071834292018000200209\&lng=es\&nrm=iso\&tlng=en

SINAGAP. (04 de Enero de 2016). Ministerio de Agricultura, Ganadería, Acuacultura y Pesca. Recuperado el 28 de Mayo de 2018, de Ministerio de Agricultura, Ganadería, Acuacultura y Pesca: http://sinagap.agricultura.gob.ec/index.php/resultados-censo-nacional/file/591-reporte-de-resultadoscenso-nacional-completo

Tello, C., Oña, C., Jarrín, M. y Viera, W. (2018). Eficacia de fungicidas para el manejo de marchitez en mora de Castilla. Quito: INIAP. Recuperado el 30 de Septiembre de 2019, de https://www.researchgate.net/publication/327252067_Eficacia_de_fungicidas_para_el_manejo_de_marchitez_en_mora_ de_Castilla

Van Driesche, R., Hoddle, M. y Center, T. (2007). Control de plagas y malezas por enemigos naturales. Washington DC, EE. UU.: USDA. Recuperado el 18 de Abril de 2018, de https://www.fs.fed.us/ foresthealth/technology/pdfs/VANDRIESCHE_CONTROL_Y_PLAGAS_WEB.pdf

Vásquez, W., Pupiales, P., Viteri, P., Sotomayor, A., Feican, C., Campaña, D. y Viera, W. (2019). Escarificación química y aplicación de ácido giberélico para la germinación de semillas cultivares de mora (Rubus glaucus BENTH). Interciencia (44): 159-164. Recuperado el 30 de Septiembre de 2019, de https://www.researchgate.net/publication/332448206_ESCARIFICACION_QUIMICA_Y_APLICACION_DE_ACIDO_GIBERELICO_PARA_LA_GERMINACION_DE_SEMILLAS_DE_CULTIVARES_DE_MORA_ Rubus_glaucus_BENTH

Villares, M., Martínez, A., Viterí, P., Viera, W., Jácome, R., Ayala, G. y Noboa, M. (2016). Manejo de plagas identificadas en el cultivo de la mora de castilla. En D. Galarza, S. Gárces, J. Velásquez, V. Sánchez y J. Zambrano, El cultivo de la mora en el Ecuador (p. 117-134). Quito: Instituto Nacional de Investigaciones Agropecuarias (INIAP).

Viteri, P., Vásquez, W., Martínez, A. V., Sotomayor, A., Mejía, P. y Brito, B. (2016). Características generales de la planta, variedades cultivadas y clones promisorios de mora. En D. Galarza, S. Garcés, J. Velásquez, V. Sánchez J. Zambrano, El cultivo de mora en el Ecuador (p. 39-54). Quito: Instituto Nacional de Investigaciones Agropecuarias (INIAP). 